\title{
Cytomegalovirus Vaccines
}

\author{
Michael A. McVoy \\ Department of Pediatrics, Virginia Commonwealth University School of Medicine, Richmond
}

An effective cytomegalovirus (CMV) vaccine could prevent the majority of birth defects caused by congenital CMV infections. Candidate vaccines in clinical evaluation include live attenuated, protein subunit, DNA, and viral-vectored approaches. Subunit approaches have focused on the CMV proteins pp65 and IE1 as important inducers of cytotoxic $\mathrm{T}$ cells and glycoprotein $\mathrm{B}(\mathrm{gB})$ as an important inducer of neutralizing antibodies. A vaccine comprised of recombinant gB protein with MF59 adjuvant reduced the incidence of primary infection by $50 \%$. Recent revelations regarding CMV entry pathways into different cell types suggest a possible course for improvement. A 5-subunit pentameric complex is uniquely required for endothelial and epithelial cell entry. Sera from naturally infected subjects contain high-potency neutralizing activities specific for this complex, whereas the gB/MF59 vaccine fails to induce comparable neutralizing activities. A vaccine's ability to induce salivary antibodies that neutralize epithelial cell entry may be especially important for preventing oral transmission as the first cells infected are presumably epithelial cells of the oral mucosa. In addition, recent evidence suggests that antibodies can inhibit postentry CMV spread between endothelial and epithelial cells. Such activities may serve to limit viral replication in tissues or impair dissemination to the placenta and fetus. Thus, inclusion of epitopes derived from the pentameric complex may provide enhanced efficacy by inducing potent neutralizing/spread-inhibiting antibodies that target virus replication in a broad spectrum of cell types. Next-generation vaccine candidates in preclinical development incorporate peptides, subunits, or multisubunit complexes representing parts or all of the pentameric complex. Approaches include peptides, recombinant proteins, DNA, replication-defective viral vectors, genetically disabled CMV, and inactivated CMV virions. The diversity of novel strategies under development engenders optimism that a successful candidate will emerge.

Keywords. cytomegalovirus; congenital infection; vaccine.

Cytomegalovirus (CMV) is among the largest and most complex of the known viruses that cause human disease. The $235-\mathrm{kb}$ genome encodes at least 165 proteins [1], but CMV vaccine research has focused on a limited number of viral proteins that dominate cellular or humoral immune responses during natural infection. The pp65 protein is a major target of the cytotoxic $\mathrm{T}$-cell response [2]. Located within the tegument

Correspondence: Michael A. McVoy, BS, PhD, PO Box 163, Department of Pediatrics, Virginia Commonwealth University School of Medicine, MCV Station, Richmond, VA 23298-0163 (mmcvoy@vcu.edu).

Clinical Infectious Diseases 2013;57(S4):S196-9

(C) The Author 2013. Published by Oxford University Press on behalf of the Infectious Diseases Society of America. All rights reserved. For Permissions, please e-mail: journals.permissions@oup.com.

DOI: 10.1093/cid/cit587 between the capsid and the viral envelope, pp65 is the most abundant protein in CMV virions. The IE1 protein is also an important cytotoxic T-cell target that is not in the virion but is abundantly expressed in cells after infection. On the virion surface and embedded in the envelope are several glycoprotein complexes that mediate host cell entry. A heterodimer comprised of glycoprotein $\mathrm{M}$ and glycoprotein $\mathrm{N}$ is believed to initiate host cell interaction by binding to heparin. A second heterodimer comprised of glycoprotein $\mathrm{H}$ and glycoprotein $\mathrm{L}(\mathrm{gH} / \mathrm{gL}$ ) may mediate receptor interactions that culminate in the triggering of conformational changes in glycoprotein $\mathrm{B}(\mathrm{gB})$ that drive fusion of the viral envelope with the target cell membrane [3]. All of these complexes are important targets for humoral immunity as they contain epitopes that bind a select class of antibodies known as neutralizing antibodies [4-7]. 
By binding to viral entry mediators, neutralizing antibodies can block cell entry and hence neutralize virus infectivity.

\section{LIVE ATTENUATED VACCINES}

Live attenuated vaccines present potential advantages for complex viruses such as CMV. By expressing a full or nearly full complement of viral antigens, they can induce both humoral and cellular immune responses that closely mimic those induced by natural infection. However, safety is a significant concern for a live CMV vaccine, especially if inadvertently administered during pregnancy. The live Towne vaccine consists of a CMV isolate that was attenuated by serial passage on cultured human fibroblasts. It has been safely administered to nearly 1000 volunteers and induces both cellular and humoral responses. When used at a low dose, the Towne vaccine failed to protect against primary maternal infection [8]. However, a higher dose was subsequently shown to be more immunogenic; efficacy of this dose has not been reported.

In an attempt to improve the immunogenicity of the Towne vaccine while retaining its safety profile, regions comprising approximately $25 \%$ of the Towne genome were replaced with corresponding sequences from the low-passage Toledo strain to produce 4 different "Towne-Toledo chimeras." Four live attenuated vaccines, each containing a different chimera, had no vaccine-related adverse events when administered to CMVseropositive volunteers but did not boost CMV immunity above preexisting levels [9]. A phase 1 trial of the 4 chimera vaccines in CMV-seronegative volunteers is in progress.

\section{SUBUNIT VACCINES}

Subunit vaccines provide potent, focused immune responses to select viral immunogens. The simplest subunit vaccines combine subunit immunogens (eg, recombinant proteins) with an adjuvant, whereas vectored delivery systems rely on genetic programming of host cells (via uptake of naked DNA or infection with replication defective viruses) to express the desired immunogen(s) in vivo. Subunit vaccine strategies have focused primarily on pp65 and IE1 to induce cellular immunity and gB to induce neutralizing antibodies. The gB/MF59 vaccine developed by Sanofi combines a recombinant soluble form of gB with MF59, an oil and water adjuvant [10]. In phase 1 trials, the gB/MF59 vaccine was both safe and highly immunogenic [10]. In a phase 2 trial, it reduced the incidence of primary infection by $50 \%$ [11]. A subsequent study showed that patients who received the $\mathrm{gB} /$ MF59 vaccine prior to renal transplantation had reduced duration of CMV viremia and required fewer days of antiviral treatment [12]. A similar vaccine comprised of recombinant $\mathrm{gB}$ protein adjuvanted with AS01 has been developed by GlaxoSmithKline. It was safe and immunogenic in a phase 1 trial.

\section{PLASMID DNA AND VIRAL-VECTORED VACCINES}

The TransVax vaccine was developed by Vical for use as a therapeutic vaccine in transplant patients. It is comprised of plasmid DNAs encoding pp65 and gB formulated with poloxamer adjuvant. In a phase 2 trial, TransVax reduced posttransplant CMV viremia in patients undergoing hematopoietic cell transplant [13]. TransVax has been licensed to Astellas Pharma Inc and has entered a phase 3 trial in hematopoietic cell transplant patients. Vical's congenital CMV vaccine, CyMVectin, is in late preclinical development. CyMVectin is comprised of plasmids that express pp65 and gB formulated with Vaxfectin, an adjuvant that promotes robust cellular and humoral responses.

AVX601 is an RNA virus-vectored vaccine developed by Alphavax. This platform uses replication-defective Venezuelan equine encephalitis virus to express $\mathrm{gB}$ and a pp65/IE1 fusion protein [14]. The vaccine has been licensed by Novartis and was evaluated in a phase 1 trial.

\section{THE PENTAMERIC COMPLEX}

The focus on $\mathrm{gB}$ as a vaccine immunogen arose from studies indicating that gB-specific antibodies comprise the majority of the neutralizing activity in CMV-seropositive sera [4]. These studies measured the ability of antibodies to neutralize virus entry into fibroblast cells. More recent studies revealed that CMV entry mechanisms are cell-type specific - whereas gB and the $\mathrm{gH} / \mathrm{gL}$ dimer are necessary for fibroblast entry, endothelial or epithelial cell entry requires 3 additional viral proteins, UL128, UL130, and UL131A, which interact with $\mathrm{gH} / \mathrm{gL}$ to form a pentameric complex [15]. That the pentameric complex is fully dispensable for fibroblast entry suggested that fibroblast-based neutralizing assays are incapable of detecting neutralizing antibodies that target epitopes unique to the pentameric complex. To address this issue, fibroblasts and epithelial cells were used to measure the neutralizing activities of CMV-seropositive sera. On average, neutralizing activities against epithelial entry were 48-fold greater than those against fibroblast entry, suggesting that a large component of neutralizing activity was missed by previous fibroblastbased assays. When epithelial neutralizing titers were measured for Towne and gB/MF59 vaccine recipients, neither vaccine came close to inducing epithelial entry neutralizing titers comparable to those induced by natural infection-gB/MF59 was 15 -fold lower, whereas Towne was 28 -fold lower [16]. Subsequent studies showed that the majority of the epithelial-specific neutralizing response is directed against epitopes within the pentameric complex [17].

These findings suggest that induction of robust epithelial/ endothelial-specific neutralizing activities may be necessary for an effective CMV vaccine. As most CMV infections are 
Table 1. Experimental Cytomegalovirus Vaccines in or Approaching Clinical Evaluation

\begin{tabular}{|c|c|c|c|c|}
\hline Type & Vaccine & Developer & Components & Status \\
\hline \multirow[t]{2}{*}{ Live attenuated } & Towne & Wistar & Whole virus & Phase 2 \\
\hline & Towne-Toledo 1, 2, 3, 4 & Aviron/Medlmmune & Whole virus & Phase 1 \\
\hline \multirow[t]{2}{*}{ Subunit protein } & gB/MF59 & Chiron/Sanofi & gB/MF59 & Phase 2 \\
\hline & GSK1492903A & GlaxoSmithKline & gB/ASO1 & Phase 1 \\
\hline \multirow[t]{2}{*}{ Subunit DNA vectored } & TransVax & Vical & gB, pp65 & Phase 2 \\
\hline & CyMVectin & Vical & gB, pp65 & Late preclinical \\
\hline Subunit viral vectored & AVX601 & Alphavax/Novartis & gB/pp65-IE1 & Phase 1 \\
\hline
\end{tabular}

acquired orally, epithelial-specific neutralizing activities in saliva could block viral transmission by preventing entry of inoculum virus into epithelial cells of the oral mucosa [18]. In cases where salivary antibodies are ineffective, serum antibodies may serve to limit CMV replication and spread within tissues or to impair dissemination to the placenta, and ultimately, the fetus. In vitro, antibodies can inhibit CMV spread between endothelial or epithelial cells but not fibroblasts [19]. Thus, antibodies that inhibit epithelial/endothelial entry/spread may be important for preventing maternal infection or, failing that, for impairing spread and/or dissemination in vivo, thereby reducing the risk of transmission to the fetus.

\section{NEW VACCINE STRATEGIES}

Vaccines designed to generate neutralizing responses that target both fibroblast and epithelial/endothelial cell entry are in preclinical development. However, the extent to which conformational and/or multisubunit-dependent epitopes dominate the "neutralizing epitome" of the pentameric complex remains unclear. That it may be necessary to represent the complete pentameric complex in its conformationally native state is suggested by a study of monoclonal antibodies isolated from naturally infected subjects: of 17 pentameric complex-specific neutralizing antibodies, all but 1 recognized multisubunit-dependent epitopes [6]. At the other end of the complexity spectrum, a UL130 subunit DNA vaccine induces epithelial-specific neutralizing responses and immunization with 2 short peptides from UL130 and UL131 induces neutralizing titers in rabbits that exceed those of most seropositive human sera [20].

A variety of vaccine approaches are under study, including simple peptides or subunits; recombinant multisubunit complexes such as $\mathrm{gH} / \mathrm{gL}$ or the complete pentameric complex; inactivated CMV virions containing native pentameric complex, gB, pp65, and other viral antigens; genetically disabled CMV expressing native pentameric complex, which potentially combines the immunogenicity of a live vaccine with the safety of a killed vaccine; replication-defective viral vectors (eg, pox, adenovirus, alphavirus, and others) expressing subunits or multisubunit complexes; and prime/boost combinations of the above.

\section{CONCLUSIONS}

Several experimental CMV vaccines have advanced to clinical evaluation (Table 1) and one, gB/MF59, has some protective efficacy. Incorporation of epitopes derived from the pentameric complex may provide additional efficacy by inducing potent neutralizing/spread-inhibiting antibodies that target virus replication in a broad spectrum of cell types. Next-generation vaccine candidates that incorporate epitopes of the pentameric complex are in preclinical development. The diversity of novel strategies under development engenders optimism that a successful candidate will emerge.

\section{Notes}

Acknowledgments. I thank Dr Stuart Adler and Dr Giovanni Nigro.

Financial support. This work was supported by grants from the National Institutes of Health.

Supplement sponsorship. This manuscript appears as part of the supplement "Prenatal Therapy of Congenital Cytomegalovirus Infection," sponsored by the Anticito Onlus Association.

Potential conflicts of interest. Author certifies no potential conflicts of interest.

The author has submitted the ICMJE Form for Disclosure of Potential Conflicts of Interest. Conflicts that the editors consider relevant to the content of the manuscript have been disclosed.

\section{References}

1. Dolan A, Cunningham C, Hector RD, et al. Genetic content of wildtype human cytomegalovirus. J Gen Virol 2004; 85(Pt 5):1301-12.

2. Wills MR, Carmichael AJ, Mynard K, et al. The human cytotoxic Tlymphocyte (CTL) response to cytomegalovirus is dominated by structural protein pp65: frequency, specificity, and T- cell receptor usage of pp65-specific CTL. J Virol 1996; 70:7569-79.

3. Wille PT, Wisner TW, Ryckman B, Johnson DC. Human cytomegalovirus (HCMV) glycoprotein $\mathrm{gB}$ promotes virus entry in trans acting as the viral fusion protein rather than as a receptor-binding protein. MBio 2013; 4:1-9.

4. Marshall GS, Rabalais GP, Stout GG, Waldeyer SL. Antibodies to recombinant-derived glycoprotein B after natural human cytomegalovirus infection correlate with neutralizing activity. J Infect Dis 1992; 165:381-4. 
5. Urban M, Klein M, Britt WJ, Hassfurther E, Mach M. Glycoprotein H of human cytomegalovirus is a major antigen for the neutralizing humoral immune response. J Gen Virol 1996; 77(Pt 7):1537-47.

6. Macagno A, Bernasconi NL, Vanzetta F, et al. Isolation of human monoclonal antibodies that potently neutralize human cytomegalovirus infection by targeting different epitopes on the gH/gL/UL128-131A complex. J Virol 2010; 84:1005-13.

7. Shimamura M, Mach M, Britt WJ. Human cytomegalovirus infection elicits a glycoprotein $\mathrm{M}(\mathrm{gM}) / \mathrm{gN}$-specific virus-neutralizing antibody response. J Virol 2006; 80:4591-600.

8. Adler SP, Starr SE, Plotkin SA, et al. Immunity induced by primary human cytomegalovirus infection protects against secondary infection among women of childbearing age. J Infect Dis 1995; 171:26-32. Erratum in J Infect Dis 1995; 171:1080.

9. Heineman TC, Schleiss M, Bernstein DI, et al. A phase 1 study of 4 live, recombinant human cytomegalovirus Towne/Toledo chimeric vaccines. J Infect Dis 2006; 193:1350-60.

10. Frey SE, Harrison C, Pass RF, et al. Effects of antigen dose and immunization regimens on antibody responses to a cytomegalovirus glycoprotein B subunit vaccine. J Infect Dis 1999; 180:1700-3.

11. Pass RF, Zhang C, Evans A, et al. Vaccine prevention of maternal cytomegalovirus infection. N Engl J Med 2009; 360:1191-9.

12. Griffiths PD, Stanton A, McCarrell E, et al. Cytomegalovirus glycoprotein-B vaccine with MF59 adjuvant in transplant recipients: a phase 2 randomised placebo-controlled trial. Lancet 2011; 377: $1256-63$.
13. Kharfan-Dabaja MA, Boeckh M, Wilck MB, et al. A novel therapeutic cytomegalovirus DNA vaccine in allogeneic haemopoietic stem-cell transplantation: a randomised, double-blind, placebo-controlled, phase 2 trial. Lancet Infect Dis 2012; 12:290-9.

14. Reap EA, Morris J, Dryga SA, et al. Development and preclinical evaluation of an alphavirus replicon particle vaccine for cytomegalovirus. Vaccine 2007; 25:7441-9.

15. Wang D, Shenk T. Human cytomegalovirus virion protein complex required for epithelial and endothelial cell tropism. Proc Natl Acad Sci U S A 2005; 102:18153-8.

16. Cui X, Meza BP, Adler SP, McVoy MA. Cytomegalovirus vaccines fail to induce epithelial entry neutralizing antibodies comparable to natural infection. Vaccine 2008; 26:5760-6.

17. Fouts AE, Chan P, Stephan JP, Vandlen R, Feierbach B. Antibodies against the $\mathrm{gH} / \mathrm{gL} / \mathrm{UL} 128 / \mathrm{UL} 130 / \mathrm{UL} 131$ complex comprise the majority of the anti-CMV neutralizing antibody response in CMV-HIG. J Virol 2012; 86:7444-7.

18. Saccoccio FM, Gallagher MK, Adler SP, McVoy MA. Neutralizing activity of saliva against cytomegalovirus. Clin Vaccine Immunol 2011; 18:1536-42.

19. Cui X, Lee R, Adler SP, McVoy MA. Antibody inhibition of human cytomegalovirus spread in epithelial cell cultures. J Virol Methods 2013; 192:44-50.

20. Saccoccio FM, Sauer AL, Cui X, et al. Peptides from cytomegalovirus UL130 and UL131 proteins induce high titer antibodies that block viral entry into mucosal epithelial cells. Vaccine 2011; 29:2705-11. 